\title{
Implicit attenuation of subsequent emotion by cognitive activity
}

\author{
Saea Iida $\cdot$ Takashi Nakao $\cdot$ Hideki Ohira
}

Published online: 27 May 2011

(C) Psychonomic Society, Inc. 2011

\begin{abstract}
The successful regulation of emotion relies heavily on executive function. Until very recently, however, the specific effects of executive function activity on other processes havereceived relatively little empirical attention.The link between executive function and emotional responses suggests that the activation of executive function may play an important role in the attenuation of emotional responses. We conducted twoexperiments to test the hypothesis that decrements in emotional responses following cognitive task performance might be caused, at least in part, by the activation of executive function.First, we sought to test whetherthe activation of executive function reducedemotional responsesimplicitly, without any volitional effort.Next, we sought to examine the link between prior activation of executivefunction and the attenuation of subsequent emotional responses, by comparing the effects of an executive-function-demanding task with the effects of a task that is equally effortful but does not engage executive function. We discuss our results in the context of the role of executive function in the attenuation of emotion.
\end{abstract}

S. Iida $\cdot$ H. Ohira $(\bowtie)$

Department of Psychology,

Graduate School of Environmental Studies, Nagoya University, Furo-cho, Chikusa-ku,

Nagoya, Aichi 464-8601, Japan

e-mail: ohira@lit.nagoya-u.ac.jp

T. Nakao

Japan Society for the Promotion of Science (JSPS), Mind, Brain Imaging and Neuroethics Institute of Mental Health Research, Royal Ottawa Health Care Group, University of Ottawa,

Ottawa, Ontario, Canada

S. Iida $(\square)$

Institute for Developmental Research, Aichi Human Service Center,

713-8 Kamiya-cho,

Kasugai, Aichi 480-0392, Japan

e-mail: iida@inst-hsc.jp
Keywords Cognitive control · Emotion

\section{Introduction}

Much previous research on emotion has been devoted to the study of the relationship between emotion andcognition. In recent years, more effort has been made to integrate the two,rather than treating them as opposing or distinct entities, byincorporating cognitive processes into models of emotionalreactions (e.g., Scherer, 2003).The cognitive processes that appear to facilitate the control of emotional reactivityinclude the regulation of attention, inhibitory control, and a group of processes referred to collectively as executive function (Fox, Henderson, \& Marshall, 2001; Ruff \& Rothbart, 1996). Some tests ofexecutive function can be used to predict emotion and self-regulatory success in sociallycomplex situations (Payne, 2005; von Hippel \& Gonsalkorale, 2005; von Hippel, Silver \& Lynch, 2000). The successfulregulation of emotion relies heavily on executive function (e.g., Banfield, Wyland, Macrae, Munte \& Heatherton, 2004; Denckla, 1996). These studies suggesta tight link between executive function and the ability to regulate emotion.

Executive function activity has often been used as a focus of manipulation in studies ofemotion regulation. For example, Erber and Tesser (1992) reported that participants who engaged in a mathematical task after watching a sad movie were less sad than participants in a control group who did not undertake the task. Erk, Abler and Walter (2006) reported that participants who engaged in an $n$-back task after anticipatory anxiety was induced by a cue depicting a schematic "frowny" or neutral "smiley" exhibited less anxiety than did a control group who did not carry out the $n$-back task. The successful inhibition of 
unpleasant emotions has been reported across a wide range of emotionregulation strategies that rely onexecutivefunction activity (e.g., Hariri, Mattay, Tessitore, Fera \& Weinberger, 2003; Liberzon et al., 2000; Monk et al., 2003).Until very recently, however, the specific effects of executive function activity alone have received little empirical attention.

These links between executive function and emotional responses suggest that the activation of executive function may be an importantcomponent of the attenuation of emotional responses. We conducted two experiments to test the hypothesis that decrements in emotional responsescould be caused in part by the activation of executive function. First, we sought to test whetherthe activation of executive function would reduce the emotional response without any volitional effort.Next, we sought to examine the link between prior activation of executivefunction and the attenuation of subsequent emotional responses. We discuss our results in the context of the role of executive function in the attenuation of emotion.

\section{Experiment 1}

Experiment 1 was designed to testwhether executive function activity implicitly attenuated emotional responses. Using a task in which no explicit instructions are given regarding the subsequent manipulation of induced emotions prevents participants from having any predictions or intentions regarding the cognitive task. That is, instruction-free tasks exclude the explicit use of emotion regulation strategies. To investigate various aspects of the effects of prior cognitive activity on emotion, we measured both self-reports and physiological responses, including heart rate (HR) and skin conductance level (SCL). We used three typical executive function tasks, the $n$-back task, the go/no-go task, and a modified version of the Wisconsin card sorting test (WCST; Grant \& Berg, 1948), all of whichrequire slightly different cognitive abilities; the $n$-back task requires the maintenance and permanent updating of relevant pieces of information in working memory, the go/ no-go task requires inhibitory control of a response, and the WCST requires switching.In addition, we used the National Aeronautics and Space Administration (NASA) Task Load Index to test whether the various cognitive tasks were equally effortful.

Method

\section{Participants}

Sixty normal healthy graduate and undergraduate students (47 male, 13 female) volunteered to take part in the study.
Participants had a mean age of 24.1 years (range, 21-28, $S D=2.1$ ), and none reported a history of major neurological or psychiatric disorders. After providing written informed consent, participants were randomly divided into three experimental groups (n-back group, go/no-go group, WCST group) and a control group.

\section{Procedure}

After the instructions for the task were provided, physiological sensors were attached to each participant. Participants underwent four testing stages within a single experimental session: baseline (10 min), cognitive task (5 $\mathrm{min})$, emotional task (10 $\mathrm{min})$, and recovery (10 $\mathrm{min})$. In the baseline stage, participants were asked to remain in a calm and relaxed state. Next, participants were instructed to engage in their respective cognitive task, followed by an emotioninduction task. In the recovery stage, participants were again asked to remain in a calm and relaxed state for $10 \mathrm{~min}$. We collected self-reports of unpleasant emotions between the stages throughout the experiment and measured physiological responses throughout the entire session.

Cognitive task In the cognitive task, a sequence of onedigit numbers was presented in a random order from among 50 even numbers and 100 odd numbers. Half of them were colored black, and the others were blue. Each number was shown for $1 \mathrm{~s}$ after an asterisk had been displayed for $1 \mathrm{~s}$ at the center of a screen as the point of gaze. For the control group, participants were asked to view the numbers passively. The $n$-back group was asked to press the target key when the number presented was identical to the number that preceded it by two numbers. Otherwise, they were to press the nontarget key. The go/no-go group was asked to press the target key if the number presented was an odd number. For the WCST group, there were two rules. First, participants were instructed to focus on the color of the number and to press the target key if the number wasblue and, otherwise, press the nontarget key. Second, participants were instructed to focus on whether the number was odd or even and press the target key if the number was odd and the nontarget key otherwise. All of the experimental groups received feedback regarding the accuracy of their keypressing. Responses were collected using an RB-730 response pad (Cedrus Corporation).

Emotional task Forty unpleasant pictures and ten neutral pictures from the International Affective Picture System (IAPS; Lang, Bradley \& Cuthbert, 1999) were used for the emotional task. In a preliminary study, the two categories significantly differed from each other with respect to their normative valence ratings $(M=2.8$ and 5.4 for unpleasant and neutral contents on a $1-9$ scale, respectively $), t(45.7)=$ 
$38.9, p<.01, r=.27$. There was no significant difference in the mean arousal levels between the two categories (5.9 and 5.6 for unpleasant and neutral contents on a 1-9 scale, respectively), $t(15.8)=2.72$, n.s., $r=.11$. Both the unpleasant and neutral images were divided into two groups and shown in a random sequence before and after a short rest, respectively. The neutral pictures were used to prevent habituation of the emotional responses to the negative pictures. Each image was shown for $1 \mathrm{~s}$, and the instruction "How negative is this image?" was presented for $2 \mathrm{~s}$. Participants were asked to evaluate the negativity of the image on a $1-5$ scale before the instruction disappeared. This task was designed to keep participants' attention directed toward the pictures. The results revealed no significant difference in the evaluation of the unpleasantness of negative pictures among the four groups, $F(3,56)=$ 2.38 , n.s., $\eta^{2}=.11$. After $10 \mathrm{~s}$, the next trial began. The responses were collected with an RB-730 response pad (Cedrus Corporation).

\section{Measures}

Self-report The unpleasant emotion induced by the emotional task was measured in terms ofthe negative affect scores in the Positive and Negative Affect Schedule (PANAS, Japanese version; Sato \& Yasuda, 2001). The negative affect scores included eight items, which were measured on a $1-5$ scale. To measure and match the task load of each cognitive task, we used the NASA Task Load

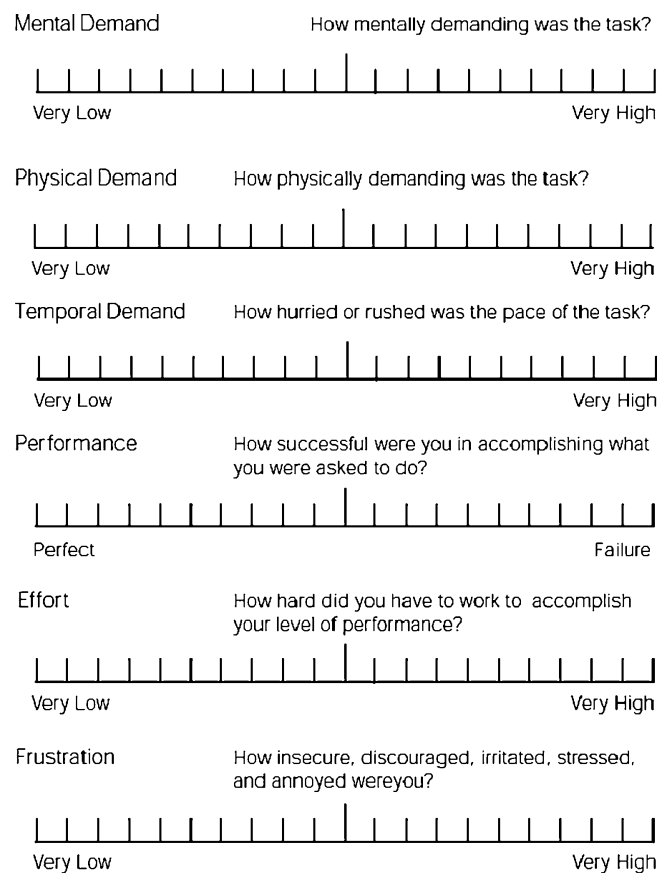

Fig. 1 NASA Task Load Index
Index (Japanese version; Haga \& Mizukami, 1996; Fig. 1 ). The NASA Task Load Index includes sixdimensions: mentaldemand, physical demand, temporal demand, performance, effort, and frustration. The use of these six subscales to compute an overall workload score has been found to reduce variability among participants, relative to a onedimensional workload rating(Hart \& Staveland, 1988). Twenty-stepbipolar scales are used to obtain ratings for these dimensions. A scorefrom 0 to 100 (assigned to the nearest 5) is obtained on each scale. An overall workload score was assessed using an unweighted average of the subscalevalues, because high correlations have been shown between weighted and unweighted scores (Byers, Bittner, \& Hill, 1989).PANAS scores were collected after each stage throughout the experiment, and NASA Task Load Index scores were collected after the entire experimental session was finished.

Autonomic measures HR was recorded using an MP-100 psychophysiological monitoring system (BioPac Systems, Santa Barbara, CA) with a 35-Hz low-pass filter and $0.5-\mathrm{Hz}$ high-pass filter. For each participant, $\mathrm{Ag} / \mathrm{AgCl}$ electrodes filled with isotonic NaClunibase electrolytes were attached to the right side of the neck and the inner surface of the left forearm for measuring an electrocardiogram. SCL was recorded using an SCL/R unit (Vega Systems), and electrodes (Vitrode P-150) were attached to the volar surface of the second phalanx of the forefinger and the middle finger of the left hand. The SCL/R unit used a 5-Hz high-pass filter, and the sampling rate was $1000 \mathrm{~Hz} . \mathrm{HR}$ and SCL were measured continuously throughout the experimental session, and the resulting data were analyzed offline using Acknowledge software (BioPac Systems, Santa Barbara, CA). Measures of HR and SCL were averaged over each experimental stage.

\section{Data analysis}

As a self-report measure, mean PANAS scores for each sampling time were calculated. For autonomic responses, the mean values of HR and SCL data were calculated for each experimental stage. We used analyses of variance (ANOVAs) for each dependent variable, with stages (baseline, cognitive task, emotional task, recovery) as a repeated measure and groups (control, $n$-back, go/no-go, WCST) as a between-participants factor. The Huynh-Feldt epsilon correction factor was used where appropriate. In cases in which a significant interaction effect or main effectwas found by ANOVA, post hoc analyses were conducted using Bonferroni tests to examine which combinations of data points differed significantly. 
Results

\section{Self-report measures}

Analysis of the PANAS scores revealed a significant interaction between stages and groups, $F(9,168)=6.06$, $p<.01, \eta^{2}=.27$. In addition, there was a significant difference between the control and experimental groups after the emotional task $(p<.01)$, such that all of the experimental groups exhibited less negative affect than did the control group (Fig. 2). Thus, the performance of all three cognitive tasks we tested inhibited the induction of a negative emotionby the emotional task, despite clear differences in specific aspects of the tasks.

Analysis of the NASA Task Load Index scores revealed a significant main effect between groups, $F(3,56)=12.75$, $p<.01, \eta^{2}=.21$. A significant difference was found between the control and experimental groups $(p<.01)$. Although all of the experimental groups showed a higher task load than did the control group, there were no significant differences among the experimental groups (Fig. 3). This indicates that each task was associated with a comparable cognitive load.

\section{Autonomic responses}

A significant interaction was foundbetween stages and groups for both HR and SCL, $F(9,168)=7.24, p<.01, \eta^{2}=.25 ; F(9$, $168)=2.03, p<.05, \eta^{2}=.20$. A significant HR difference was found between the control and experimental groups in the emotional task $(p<.01)$, and a significant difference in SCL was found in the cognitive task. The simple main effect of the stage factor for each group was significant [control, $F$ $(3,168)=10.03, p<.01, \eta^{2}=.19 ; n$-back, $F(3,168)=6.73$, $p<.01, \eta^{2}=.17$; go/no-go, $F(3,168)=9.42, p<.01, \eta^{2}=$ .21 ; WCST, $F(3,168)=8.78, p<.01, \eta^{2}=.20$; Figs. 4 and 5]. This finding indicated that in the experimental groups, significant differences were found between the cognitive task and the other stages $(p<.05)$. In addition, in

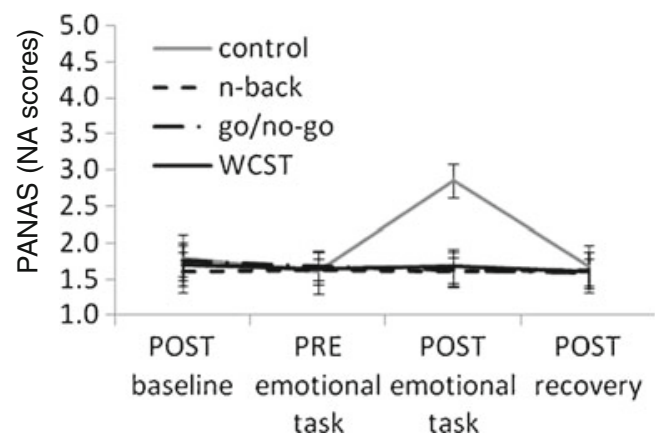

Fig. 2 Self-reports of negative emotion at post-baseline, pre/postemotional task, and post-recovery for the each group. Error bars indicate standard errors

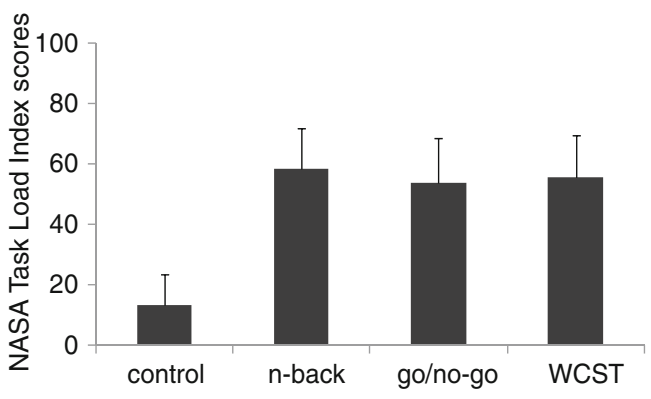

Fig. 3 Overall workload score of the NASA Task Load Index scores for each task. Error bars indicate standard errors

the control group, there were significant differences between the emotional task stage and the baseline $(p<.01)$ and cognitive task $(p<.01)$ stages. Thus, regardless of the nature of the cognitive task, performance of a cognitive task attenuated the increase in HR in the emotional task.There was no significant difference in SCL between the control group and experimental groups in the emotional task. However, although the control group showed a significant increase in SCL in the emotional task, the experimental groups tended to show a decrease in SCL, regardless of the nature of the cognitive task.

\section{Discussion}

The results were consistent with the notion that the activation of executive function attenuatesemotional responses without any volitional effort. As compared with the control group, HR during the emotional task and selfreports of negative emotions were similarly reduced in all three experimental groups. As compared with the SCL during the cognitive task, experimental groups showed significantly lower SCL during the emotional task, even though control group showed significantly higher SCL during the emotional task. That is, cognitive activity appeared to successfully attenuatesubsequent emotion in a similar way regardless of its nature.

It must be acknowledged that the present experiment included nodirect measures of the enhancement of executive function and is, thus, unable to provide directevidence

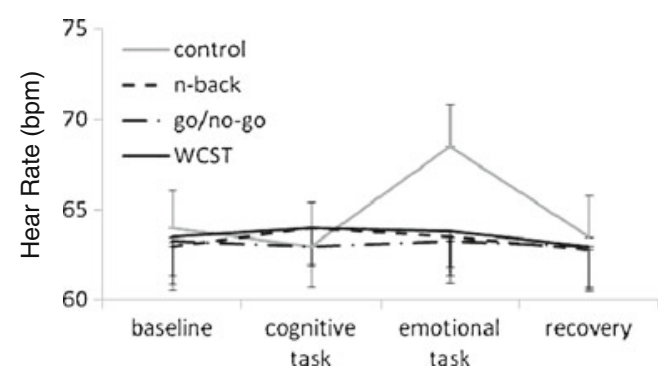

Fig. 4 Heart rate in the experimental groups at baseline, cognitive task, emotional task and recovery. Error bars indicate standard errors 


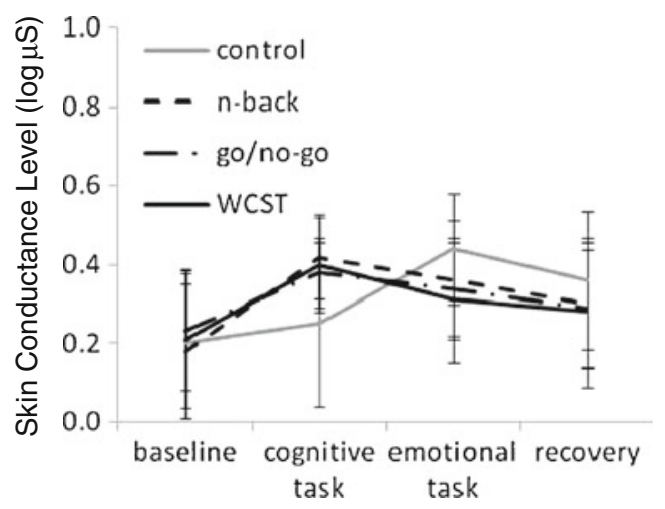

Fig. 5 Skin conductance in the experimental groups at baseline, cognitive task, emotional task and recovery. Error bars indicate standard errors

that activated executive function implicitly attenuates subsequent emotional responses.Although our behavioral results support the notion that cognitive activity attenuates unpleasant emotion, it is important to consider possible alternativeinterpretations of the results.

Onepossible alternative explanation of our findings is related to cognitive fatigue or resource consumption (e.g., Parasuraman, 1998; Wickens, 1984). The conservation of resources (CORs) theory (Hobfoll, 1989) has been used to explain the attenuation of emotion. According to this theory, stressful events lead to emotional exhaustion. The experienceof emotions and stress are known to beaccompanied by a physiological state of arousalinvolving the endocrine system (release of hormones)and the autonomic nervous system (increased HR, breathing, blood pressure, SCL; Lazarus, 1999). While in this state of arousal, the body convertspsychophysiological resources(Friedman \& Thayer, 1998; Thayer \&Friedman, 1997) into energy to respond to the current situation. Thus, in this study, the attenuation of subsequent emotionmay have been caused by a lack of resources.

\section{Experiment 2}

Experiment 2 investigated whether the consumption of resources alonecould account for the attenuation of subsequent emotion we observed in the first experiment. The SCL results in Experiment 1 indicated that the cognitivetask performancerequiredat least some psychophysiological resources. Hence, it is possible that any tasks requiringresources required for the induction of a subsequent emotional state, not just cognitive tasksspecifically, should have the same effectson subsequent emotional reactivity. To test this possibility, inExperiment 2, we compared the effect of anexecutive function task with that of anonexecutive handgrip-squeezing task. We chosehandgrip squeezing as a task that did not require executive function, but any other task consuming the same level of resources would be similarly appropriate. Althoughthe notion of a psychological energy resource is a highlyconvenient metaphor that explains a broad range of empiricalfindings, the precise nature of the energy source in this context has not been clarified. To investigate various aspects of such resources, the level of resource consumption was measured for autonomic responses and the NASA Task Load Index, as in Experiment 1. Task load is a term that represents the cost, for a participant, of accomplishing task requirements (e.g., fatigue, stress, illness; Hart, 2006; Hart \&Staveland, 1988). Therefore, ifprior resourceconsumption causes the attenuation of subsequent emotion, we would expect the same results in the executive function task group and a handgrip-squeezing group. On the other hand, if prior activation of executive function, specifically, is critical for the attenuation of subsequent emotion,this effectshould beseen in the cognitive task group,but not in the handgrip-squeezinggroup.

\section{Method}

\section{Participants}

Forty-five normal healthy graduate and undergraduate students (30 male, 15 female) volunteered to take part in the study, with amean age of 25.3 years (range, $21-29 ; S D=3.4$ ). After providing written informed consent, participants were randomly divided into three groups (cognitive task group, noncognitive task group, and control group).

\section{Procedure}

After instructions regardingthe task were provided, physiological sensors were attached to each participant. Participants underwent four testing stages within a single experimental session: baseline (10 min), cognitive/noncognitive task (5 min), emotional task (10 min), and recovery (10 min). In the baseline stage, participants were asked to remain in a calm and relaxed state. The cognitive task group and noncognitive task group were then asked to perform their respective tasks, and the control group was again asked to remain in a calm and relaxed state. After completing the first task (or rest period), all participants undertookan emotional task. In the recovery stage, participants were againasked to remain in a calm and relaxed state for $10 \mathrm{~min}$. We collected self-reports of unpleasant emotion between each stage and measured physiological responses throughout the entire session.

Cognitive task A sequence of one-digit numbers was presented in a random order. Each number was shown for $1 \mathrm{~s}$ afteran asterisk (fixation point) had been presented at 
the center of a screenfor 1 s.The cognitive task group then completed a 2-back task, in which they had to press the target key when the number presented was identical to the first of the two previous numbers. Otherwise, they were to press the nontarget key. Responses were collected using an RB-730 response pad (Cedrus Corporation).

Noncognitive task The noncognitive task groupwas instructed to squeeze a $15-\mathrm{kg}$ handgrip of a type that is commonly used to train hand muscles. The handgrip consists of two handles connected by a wound spring and is designed to build muscles in the forearm. Individuals grasp the handgrip, which brings the handles together and compresses the spring, thereby creating resistance. Participants were asked to squeeze the handgrip once every $2 \mathrm{~s}$ for $5 \mathrm{~min}$.

Emotional task The emotional task was the same as in Experiment 1 . There were no significant differences among the three groups in the evaluation of the unpleasantness of negative pictures, $F(2,42)=1.77$, n.s., $\eta^{2}=.10$, as in Experiment 1.

\section{Measures}

We used essentially the same measures as in Experiment 1 for both self-reports and autonomic responses. In the selfreport measure, the unpleasant emotion induced by the emotional task was measured in terms ofnegative affect scores onthe PANAS. We used the NASA Task Load Indexto measure the mental load of each task.PANAS scores were collected after each stage throughout the experiment, and the Task Load Index was collected after the experiment session was finished. For autonomic responses, HRand SCL were recorded, as in Experiment 1. HR and SCL were measured continuously throughout the experimental session, and the data were analyzed offline using Acknowledge software (BioPac Systems, Santa Barbara, CA, USA). Measures of HR and SCL were averaged over each experimental stage.

\section{Data analysis}

For self-reportedvalues, the mean PANAS scores for each sampling periodwere calculated. For autonomic responses, the mean values of HR and SCL data were calculated for each experimental stage. We conducted ANOVAson each dependent variable, with stage (baseline, cognitive/noncognitive task, emotional task, recovery) as a repeated measure and group (control, cognitive task, noncognitive task) as a between-participants factor. The Huynh-Feldt epsilon correction factor was used where appropriate. When a significant interaction effect or main effect was found inan ANOVA, post hoc analyses using Bonferroni tests were conducted to examine which combinations of data exhibited significant differences.

Results

\section{Self-reports}

Regarding the PANAS scores, a significant interaction was observed between stage and group, $F(6,126)=4.56$, $p<.01, \eta^{2}=.24$. Significant differenceswere found between the cognitive task group and the control $(p<.01)$ and noncognitive task $(p<.01)$ groups after the emotional task, such that thecognitive task group showed significantly less negative affect than did the noncognitive task and control groups (Fig. 6). This indicated that the cognitive task, but not the noncognitive task, attenuated negative emotion.

Task Load Index scores revealed a significant main effect of group, $F(2,42)=67.82, p<.01, \eta^{2}=.37$. Significant differences were found between the control group and the cognitive task $(p<.01)$ and noncognitive task $(p<.01)$ groups. While the cognitive task and noncognitive task groups showed a higher mental load than did the control group, there was no significant difference between the cognitive and noncognitive task groups (Fig. 7). This result indicated that the mental load for each task was well controlled.

\section{Autonomic responses}

Significant interactions between stages and groups were seen for both HR and SCL, $F(6,126)=6.83, p<.01, \eta^{2}=.21 ; F(6$, $126)=4.17, p<.01, \eta^{2}=.16$. In the cognitive/noncognitive task stage, significant differences were found between the control group and the cognitive task (HR, $p<.05$; SCL, $p<$ $.01)$ and noncognitive task (HR, $p<.01$; SCL, $p<.01$ ) groups, for both HR and SCL. In the emotional task, there were significant differences on both measures between the

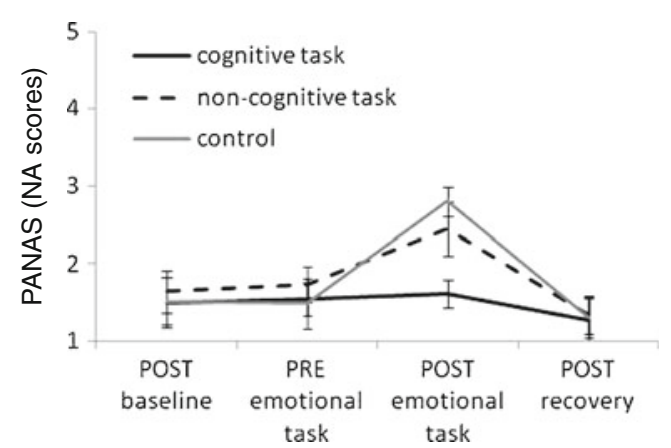

Fig. 6 Self-reports of negative emotion at post-baseline, pre/postemotional task, and post-recovery for the each group. Error bars indicate standard errors 


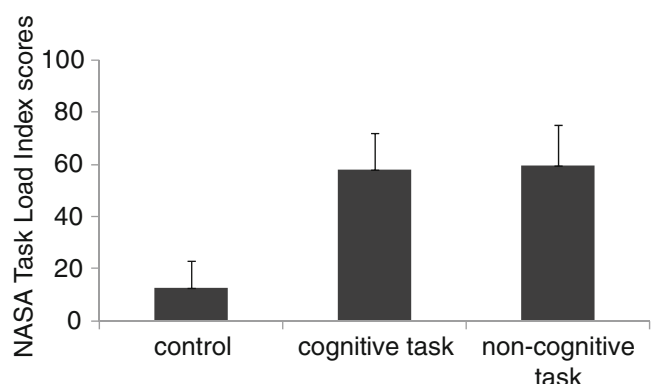

Fig. 7 Overall workload score of the NASA Task Load Index scores for each task. Error bars indicate standard errors

cognitive task group and the control (HR, $p<.05$; SCL, $p<.05$ ) and noncognitive task (HR, $p<.05$; SCL,n.s.) groups (Figs. 8 and 9). These results indicate that the noncognitive task group, like the cognitive task group, showed an enhanced autonomic response during the cognitive/noncognitivetask. However, in the emotional task, the noncognitive task did not attenuate emotional responses.

\section{Discussion}

This experiment investigated whether or notthe consumption of resources was the critical factor in causing the attenuation of subsequent emotion. Although the cognitive task group exhibited results similar to those in Experiment1, neither physiological responses nor subjective negative emotions were attenuated in the noncognitive task group. These findings indicate that the CORs theory cannot explain the effect of executive function on subsequent emotion.

\section{General discussion}

The present investigation was designed to test the hypothesis that the activation of executive function may be an important component in the attenuation of emotional responses. Both of the present experiments provided evidence supporting this notion. Experiment 1 revealed that engagement in an executive function task can implic-

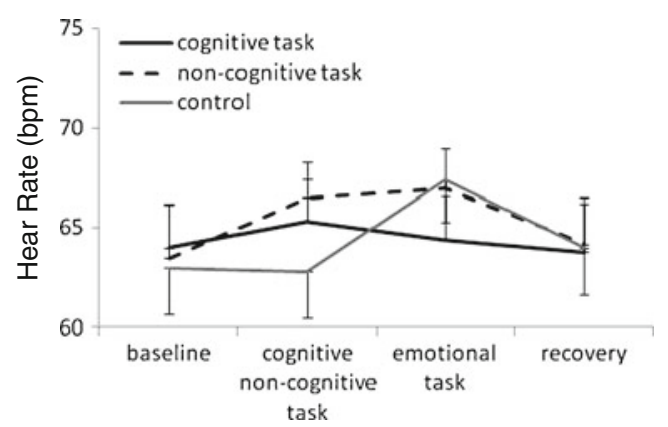

Fig. 8 Heart rate in the experimental groups at baseline, cognitive task, emotional task and recovery. Error bars indicate standard errors

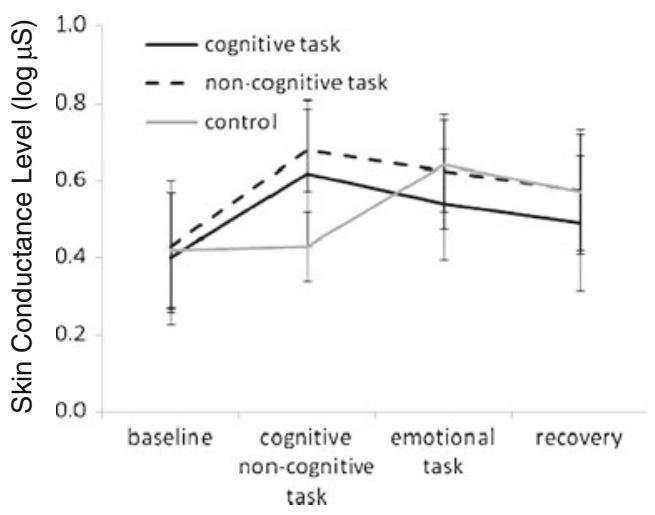

Fig. 9 Skin conductance in the experimental groups at baseline, cognitive task, emotional task and recovery. Error bars indicate standard errors

itly attenuate subsequent emotion. However, there are at least two possible explanations for this phenomenon. First, a long-term priming effect may have played a role, since practice with a working memory task can improve performance and modify underlying patterns of neural activation (e.g., Browning, Holmes, Murphy, Goodwin \& Harmer, 2010; Mowbray \& Rhoades, 1959; Woodworth, 1938). When participants practice a task, their performance often improves, either because they become more efficient at applying their initial strategy or because they learn to employ a new strategy (Jonides, 2004). Acquiring greater skill using an initial strategy is thought to recruit a similar network of brain regions after practice (Chein \& Schneider, 2005; Kelly \& Garavan, 2005). Executive function refers to attention shifting, working memory, and inhibitory control cognitive processes that are involved in planning, problemsolving, and goal-directed activities (Miyake et al., 2000). Thus, in the present study,a previous executive function task was thought to modulate the way participants process subsequent stimuli, such that a negative mood induction stimulus never elicits an emotional response in the first place. For example, Wadlinger and Isaacowitz (2011) reviewed evidence that regulating attention is a critical component of the emotion regulation process. There is evidence that prior cognitive activity can modulate attention deployment to subsequent emotional stimuli or broaden attentional capacity, as applied in various attentional training programs (e.g., See, MacLeod \& Bridle, 2009; Sohlberg \& Mateer, 1987), without repeated training. Another possible explanation is the CORs theory. According to this theory, prior cognitive activity is thought to reduce psychophysiological resources and disrupt resources for subsequent emotion elicitation. Experiment 2 examined whether the attenuation of subsequent emotion was caused by the priming effect or a lack of resources due to a prior executive function task. Although the cognitive task group showed results similar to those in Experiment 1, the 
noncognitive task group did not. These findings indicate that the activation of executive function is a critical factor in the implicit attenuation of subsequent emotion. Further research is required to clarify the mechanism by which activated executive function affects subsequent emotion.

Several recent studies have shown increased prefrontal and parietal activity in brain activity following a single session of cognitive training (Browning et al., 2010; Olesen, Westerberg \& Klingberg, 2004) and thepotential involvement of the orbitofrontal cortex, especially lateraland medial portions, lateral and ventromedial portions of the prefrontalcortex, and the basal ganglia in implicit emotion regulation. As was noted in Mauss, Bunge and Gross (2007) review, these regionshave been implicated in emotion regulation, cognition-emotion interactions, topdown direction of attention in response to negative emotionalstimuli, and encoding of affective expectations in relation to conditionedstimuli (Beer, Heerey, Keltner, Scabini \& Knight, 2003; Davidson, 2002; Elliott, Dolan \& Frith, 2000; Gottfried, O'Doherty \& Dolan, 2003; Hamann, Ely, Hoffman \& Kilts, 2002; Lieberman, 2000; Rolls, 2000). These results also support our interpretation and the possibility that the recruitment of executive function plays an important role in the attenuation of emotion.

The measurement of energy resources was an important limitation in the present study. We used the NASA Task Load Index and SCL as indices of resource consumption, whichare considered two of the most reliable scales for measuring the use of limited attentional resources by cognitive activity and self-regulation (e.g., Harris, Hancock, Authur \& Caird, 1995; Liu \& Wickens, 1994; Miyake, 2001; Rubio, Diaz, Martin \& Puente, 2004). However, as was noted above, the notion of energy resources constitutes a convenient metaphor that explains a broad range of empirical findings, but the precise nature of such an energy source has not been clarified. In the present study,engaging in the handgrip task exhibited no effect on the subsequent emotion reported by the noncognitive task group. However, Voh et al. (2010) reported that the handgrip task was associated with an inability to regulate subsequent emotion, possibly because of the conflict caused by the difference in the amount of resource consumption. Voh et al. intended for the handgrip task to deplete resources, while we sought a noncognitive task that consumed the same amount of resources as cognitive activity consumed, using a slightly different handgrip task. However, additional measurements (e.g., pupil dilation) are required toresolve this issue in more detail, focusing on the role of resources in the context of self-regulation.

Regarding future investigations, the present study revealed a new strategy for the implicit regulation of emotion, involving engagement in a cognitive task before an unpleasant emotion is elicited.In addition, we demon- strated that attenuation of unpleasant emotion requires the activation of executive function and is independent of the nature of the cognitive task and how the participant engages in the task. This new strategy is simple enough to also be used for anticipatory emotion regulation or training of the ability to regulate emotion. It was recently reported that executive function can be trained by engaging in specific kinds of cognitive tasks (e.g., Fisher \& Happé, 2005; Olesen et al., 2004). If executive function is a critical factor in emotion regulation, the routine application of this novel strategy may be used to strengthen executive function and the ability to regulate emotion. Further investigations of this new strategy are required,not only to identify the precise mechanisms of emotion attenuation, but also to identify possible applications for this strategy in daily life.

Author notes The authors wish to thank Naho Ichikawa at the Stanford University, for encouragement, support, and helpful discussion. The authors would like to extend their thanks to the editor and two anonymous reviewers for their carefully considered feedback on a previous draft of this manuscript.

\section{References}

Banfield, J., Wyland, C. L., Macrae, C. N., Munte, T. F., \& Heatherton, T. F. (2004). Thecognitive neuroscience of selfregulation. In R. F. Baumeister \& K. D. Vohs (Eds.), Thehandbook of self-regulation (pp. 62-83). New York, NY: Guilford.

Beer, J. S., Heerey, E. A., Keltner, D., Scabini, D., \& Knight, R. T. (2003). The regulatoryfunction of self-conscious emotion: Insights from patients with orbitofrontal damage. Journal of Personality and Social Psychology, 85, 594-604.

Browning, M., Holmes, E. A., Murphy, S. E., Goodwin, G. M., \& Harmer, C. J. (2010). Lateralprefrontal cortex mediates the cognitive modification of attentional bias. Biological Psychiatry, 67, 919-925.

Byers, J. C., Bittner, A. C., \& Hill, S. G. (1989). Traditional and raw task load index (TLX) correlations: Are paired comparisons necessary? In A. Mital (Ed.), Advances in industrial ergonomics and safety (Vol. 1, pp. 481-485). London: Taylor \& Francis.

Chein, J. M., \& Schneider, W. (2005). Neuroimaging studies of practice-related change: fMRI and meta-analytic evidence of a domain-general control network for learning. Cognitive Brain Research, 25, 607-623.

Davidson, R. J. (2002). Anxiety and affective style: Role of prefrontal cortex and amygdala. Biological Psychiatry, 51, 68-80.

Denckla, M. R. (1996). Research on executive function in a neurodevelopmental context: Application of clinical measures. Developmental Neuropsychology, 12, 5-15.

Elliott, R., Dolan, R. J., \& Frith, C. D. (2000). Dissociable functions in the medial and lateralorbitofrontal cortex: Evidence from human neuroimaging studies. Cerebral Cortex, 10, 308-317.

Erber, R., \& Tesser, A. (1992). Task effort and the regulation of mood: The absorption hypothesis. Journal of Experimental Social Psychology, 28, 339-359.

Erk, S., Abler, B., \& Walter, H. (2006). Cognitive modulation of emotion anticipation. European Journal of Neuroscience, 24, $1227-1236$. 
Fisher, N., \& Happé, F. (2005). A training study of theory of mind and executive function in children with autism spectrum disorders. Journal of Autism and Developmental Disorders, 35, 757-771.

Fox, N. A., Henderson, H. A., \& Marshall, P. J. (2001). The biology of temperament: An integrative approach. In C. A. Nelson \& M. Luciana (Eds.), Handbook of developmental cognitive neuroscience (pp. 631-646). Cambridge, MA: MIT Press.

Friedman, B. H., \& Thayer, J. F. (1998). Anxiety and autonomicflexibility: A cardiovascular approach. Biological Psychology, 49, 303-323.

Gottfried, J. A., O'Doherty, J., \& Dolan, R. J. (2003). Encoding predictive reward value in humanamygdala and orbitofrontal cortex. Science, 301, 1104-1107.

Grant, D. A., \& Berg, E. A. (1948). A behavioural analysis of degree of reinforcement and ease of shifting to new responses in a Weigl-type card sorting problem. Journal of Experimental Psychology, 38, 404-411.

Haga, S., \& Mizukami, N. (1996). Japanese version of NASA Task Load Index: Sensitivity of its workload score to difficulty of three different laboratory tasks. Japanese Journal of Ergonomics, 32, $71-79$.

Hamann, S. B., Ely, T. D., Hoffman, J. M., \& Kilts, C. D. (2002). Ecstasy and agony: Activationof human amygdala in positive and negative emotion. Psychological Science, 13, 135-141.

Harris, W. C., Hancock, P. A., Authur, E. J., \& Caird, J. K. (1995). Performance, workload, and fatigue changes associated with automation. InternationalJournal of Aviation Psychology, 5, $169-185$

Hariri, A. R., Mattay, V. S., Tessitore, A., Fera, F., \& Weinberger, D. R. (2003). Neocortical modulation of the amygdala response to fearful stimuli. Biological Psychiatry, 53, 494-501.

Hart, S. G. (2006). NASA-Task Load Index (NASA-TLX); 20 yearslater. In Proceedings of the Human Factors and Ergonomics Society50th Annual Meeting (pp. 904-908). Santa Monica, CA: Human Factors\& Ergonomics Society.

Hart, S. G., \& Staveland, L. E. (1988). Development of NASA-TLX (Task Load Index): Results of empirical and theoretical research. In P. A. Hancock \& N. Meshkati (Eds.), Human mental workload (pp. 139-183). Amsterdam: Elsevier.

Hobfoll, S. E. (1989). Conservation of resources: A new approach at conceptualizing stress. American Psychologist, 44, 513-524.

Jonides, J. (2004). How does practice makes perfect? Nature Neuroscience, 7, 10-11.

Kelly, A. M., \& Garavan, H. (2005). Human functional neuroimaging of brain changes associated with practice. Cerebral Cortex, 15, 1089-1102.

Lang, P. J., Bradley, M. M., \& Cuthbert, B. N. (1999). The International Affective Pictures System (IAPS):Technical Manual and Affective Ratings. Gainesville: University of Florida, Center for Research in Psychophysiology.

Lazarus, R. S. (1999). Stress and emotion. New York, NY: Springer.

Liberzon, I., Taylor, S. F., Fig, L. M., Decker, L. R., Koeppe, R. A., \& Minoshima, S. (2000). Limbic activation and psychophysiologic responses toaversive visual stimuli. Interaction with cognitive task. Neuropsycho-pharmacology, 23, 508-516.

Lieberman, M. D. (2000). Intuition: A social cognitive neuroscience approach. PsychologicalBulletin, 126, 109-137.

Liu, Y. L., \& Wickens, C. D. (1994). Mental workload and cognitive task Automaticity:An evaluation of subjective and timeestimation metrics. Ergonomics, 37, 1843-1854.

Mauss, I. B., Bunge, S. A., \& Gross, J. J. (2007). Automatic emotion Regulation. Social andPersonality Psychology Compass, 1, 146-167.
Miyake, S. (2001). Multivariate workload evaluation combining physiological and subjective measures. International Journal of Psychophysiology, 40, 233-238.

Miyake, A., Friedman, N. P., Emerson, M. J., Witzki, A. H., Howerter, A., \& Wager, T. (2000). The unity and diversity of executive functions and their contributions to complex "frontal lobe" tasks: A latent variable analysis. Cognitive Psychology, 41, 49-100.

Monk, C. S., McClure, E. B., Nelson, E. E., Zarahn, E., Bilder, R. M., Leibenluft, E., et al. (2003). Adolescent immaturity in attentionrelated brain engagement toemotional facial expressions. NeuroImage, 20, 420-428.

Mowbray, G., \& Rhoades, M. (1959). On the reduction of choicereaction times with practice. Quarterly Journal of Experimental Psychology, 11, 16-23.

Olesen, P. J., Westerberg, H., \& Klingberg, T. (2004). Increased prefrontal and parietal activity after training of working memory. Nature Neuroscience, 7, 75-79.

Parasuraman, R. (1998). The attentive brain. Cambridge, MA: MIT Press.

Payne, B. K. (2005). Conceptualizing control in social cognition: How executive functioning modulates the expression of automatic stereotyping. Journal of Personality and Social Psychology, 89, $488-503$.

Rolls, E. T. (2000). The orbitofrontal cortex and reward. Cerebral Cortex, 10, 284-294.

Rubio, S., Diaz, E., Martin, J., \& Puente, J. M. (2004). Evaluation of subjectivemental workload: A comparison of SWAT, NASATLX, and Workload Profile. Applied Psychology, 53, 61-86.

Ruff, H. A., \& Rothbart, M. K. (1996). Attention in early development: Themes and variations. NewYork: Oxford University Press.

Sato, A., \& Yasuda, A. (2001). Development of the Japanese version of Positive and Negative Affect Schedule (PANAS) scales. Japanese Journal of Personality, 9, 138-139.

Scherer, K. R. (2003). Introduction: Cognitive components of emotion. In R. J. Davidson (Ed.), Handbook of affective sciences (pp. 563-571). Cary, NC: Oxford University Press.

See, J., MacLeod, C., \& Bridle, R. (2009). The reduction of vulnerability through the modification of attentional bias: A real world study using a home-based cognitive bias modification procedure. Journal of Abnormal Psychology, 118, 65-75.

Sohlberg, M., \& Mateer, C. (1987). Effectiveness of an attentiontraining program. Journal of Clinical and Experimental Neuropsychology, 9, 117-130.

Thayer, J. F., \& Friedman, B. H. (1997). The heart of anxiety: Adynamical systems approach. In A. Vingerhoets (Ed.), The(non)expression of emotions in health and disease. Amsterdam: Springer.

vonHippel, W., \& Gonsalkorale, K. (2005). "That is bloody revolting!" Inhibitory control of thoughts better left unsaid. PsychologicalScience, 16, 497-500.

vonHippel, W., Silver, L. A., \& Lynch, M. E. (2000). Stereotyping againstyour will: The role of inhibitory ability in stereotyping and prejudiceamong the elderly. Personality and Social Psychology Bulletin, 26, 523-532.

Wadlinger, H. A., \& Isaacowitz, D. M. (2011). Fixing our focus: Training attention to regulate emotion. Personality and Social Psychology Review, 15, 75-102.

Wickens, C. D. (1984). Processing resources in attention. In R. Parasuraman \& D. R. Davies (Eds.), Varieties of attention (pp. 63-102). Orlando, FL: Academic Press.

Woodworth, R. S. (1938). Experimental psychology. New York: Holt, Rinehart \& Winston. 